\title{
Impact of Recent Job Loss on Sleep, Energy Consumption and Diet
}

Salma Batool-Anwar, M.D., M.P.H.

Candace Mayer

Patricia L. Haynes, Ph.D.

Yilin Liu

Cynthia A. Thomson, Ph.D., RDN

Stuart F. Quan, M.D.

Division of Sleep and Circadian Disorders, Brigham and Women's Hospital, Harvard Medical School, Boston, MA; Mel and Enid Zuckerman College of Public Health, University of Arizona, Tucson, AZ

Running Head: Job Loss, Sleep and Energy Consumption

Correspondent: Salma Batool-Anwar

Division of Sleep and Circadian Disorders, Brigham and Women's Hospital

221 Longwood Ave.

Boston, MA 02115

Email: sbatool@bics.bwh.harvard.edu 


\section{Abstract}

To examine how sleep quality and sleep duration affect caloric intake among those experiencing involuntary job loss.

Methods

Adequate sleep and self reported dietary recall data from the Assessing Daily Activity Patterns through Occupational Transitions (ADAPT) study was analysed. Primary sleep indices used were total sleep time, time spent in bed after final awakening, and sleep quality as measured by the Daily Sleep Diary (DSD). Mean Energy consumption (MEC) was the primary nutritional index. Secondary indices included diet quality using the Health Eating Index 2015 (HEI), and self-reported intake of protein, carbohydrates and fats.

\section{Results}

The study participants were comprised mainly of women $(61 \%)$ and non-Hispanic white. The participants had at least 2 years of college education and mean body mass index of $30.2 \pm 8.08\left(\mathrm{~kg} / \mathrm{m}^{2}\right)$. The average time in bed was $541.8 \pm 77.55$ minutes and total sleep time was $461.1 \pm 56.49$ minutes. Mean sleep efficiency was $91 \pm 6 \%$, self-reported sleep quality was $2.40 \pm 0.57(0-4$ scale, $4=$ very good), and minutes earlier than planned morning awakening were 14.36 \pm 24 .15. Mean HEI score was 47.41 \pm 10.92 . Although the MEC was below national average for both men and women, male sex was associated with higher MEC. In a fully adjusted model sleep quality was positively associated with MEC.

\section{Conclusion}

Daily overall assessments of sleep quality among recently unemployed persons were positively associated with mean energy consumption. Additionally, the diet quality of unemployed persons was found to unhealthier than the average American and consistent with the relationship between poor socioeconomic status and lower diet quality. 


\section{Introduction}

Obesity is a major public health concern; $38.3 \%$ of women and 34.3 of men ${ }^{1}$ in the United States are obese. It is not only the result of low physical activity and overconsumption of high energy yielding foods, socioeconomic status also plays a major role ${ }^{2}$. Obesity disproportionately affects people of lower socioeconomic status (SES) in part because their limited financial resources result in consumption of calorically dense unhealthy food. This contributes to the risk of developing obesity ${ }^{3}$.

A major determinant of SES is employment status. Unemployment is one indicator of a reduced SES and is associated with higher levels of stress. Unemployment includes involuntary job loss which is an important disruptive life event. It can cause additional unanticipated psychological and economic stress with the former afflicting women disproportionately ${ }^{4}$. Involuntary job loss is positively associated with greater symptoms of depression ${ }^{5}$, disruptions in daily routine changes and poor sleep quality. Unemployment has been shown to be positively associated with obesity ${ }^{6,7}$. In one study, women were more likely to be diagnosed with obesity after involuntary job loss ${ }^{8}$.

Sleep is another factor that affects obesity risk. Lower sleep quality and reductions in sleep duration have been shown to increase food intake resulting in becoming overweight or obese ${ }^{9}$. Sleep deficiency can change the secretory pattern of leptin and ghrelin leading to hunger and a craving for calorically dense food ${ }^{10,11}$.

There are no prior studies that have investigated the impact of whether sleep quality or sleep duration influences caloric consumption in those that have experienced involuntary job loss. The Assessing Daily Activity Patterns through Occupational Transitions (ADAPT) Study is an ongoing longitudinal cohort study of individuals who have suffered involuntary job loss. We analyzed cross sectional data from the baseline assessment of the ADAPT study and hypothesized that disrupted, short sleep would be associated with increased energy intake among these individuals.

\section{Methods}

\section{Participants}

Study participants were part of the on-going ADAPT Study, an 18-month longitudinal study examining changes in sleep, social rhythms, and obesity following an involuntary job loss ${ }^{12}$. The study protocol and recruitment strategy have been described in detail previously. Briefly, all individuals who applied for unemployment insurance (UI) in the greater Tucson, Arizona and surrounding areas 
between October 2015 and December 2018 received study recruitment flyers within their UI intake packets. Interested individuals contacted study staff and completed phone screens assessing exclusion criteria; potentially eligible individuals were then scheduled for in-person screening visits. Individuals were eligible if they had experienced an involuntary job loss within 90 days of study enrollment, had been with their employer for at least six months, were currently employed less than 5 hours per week and did not complete any night shift work within the last 30 days. During the in-person screening, participants provided written informed consent, as well as information about their demographics, employment and medical history. They also were screened for homelessness, existing physiological and mental health conditions, substance abuse, and major sleep diagnoses which could interfere with social rhythms and sleep patterns. Those who passed screening completed validated mental health and sleep diagnostic interviews. An overnight at-home screening for sleep apnea was performed utilizing the ApneaLink Plus ${ }^{\mathrm{TM}}$ (ResMed, San Diego, CA) to exclude moderate sleep apnea as a cause of sleep disruption.

Data used in this analysis originated from the study's baseline visit. Of the 446 adults who provided written consent, 191 participants met eligibility criteria and completed a baseline assessment visit, including an at-home data collection period lasting two weeks. Participants were considered for the current analysis if there was an acceptable assessment of sleep and diet on their sleep diaries and dietary recalls respectively for analysis. However, 8 participants were excluded as outliers because their mean energy consumption (MEC) was significantly less than commonly reported norms ${ }^{13,14}$. Descriptive statistics for the study sample are reported in Table 1.

\section{Measures}

\section{Demographic and Anthropometric}

Age, ethnicity and biological sex were collected during the initial interview. Height and weight were measured to calculate the body mass index $\left(\mathrm{BMI} \mathrm{kg} / \mathrm{m}^{2}\right)$.

\section{Diet Assessment}

During the two-week, at-home baseline data collection period, participants completed up to three 24-hour dietary recalls administered by trained diet assessors at the Behavioral Measurements and Interventions Shared Resource of the University of Arizona Cancer Center utilizing the gold-standard USDA Multi-pass Dietary recall ${ }^{15}$ and the Nutrient Database System of the University of Minnesota for nutrient analysis ${ }^{16}$ These interviews were supported by the Remote Food Photography Method ${ }^{17}$, in which participants took pictures of all food and beverages prior to consumption, as well as after they had finished eating and drinking. Photos were used to review recall as a final verification of the multi-pass data. The diet recalls provided information on the types and quantity of food, including energy and nutrient values. At least 3 dietary recalls were completed by 172 participants (95.6\% of the cohort). The primary index for this analysis was MEC (kcal). Secondary indices included diet quality estimated using the Health Eating Index 2015 (HEI) ${ }^{18}$ using standardized approaches to score, and self-reported intake of protein, carbohydrates and fats. 


\section{$\underline{\text { Sleep Measures }}$}

Sleep variables of interest were measured using the valid and reliable Daily Sleep Diary (DSD) ${ }^{19}$, the recommended subjective sleep assessment instrument of the insomnia research consensus panel. Upon wakening from their sleep, participants completed the DSD via mobile application. The DSD was completed at least 15 times by 170 participants with only 3 participants completing less than 4 days of diary data. The primary indices of interest were total sleep time (TST) and minutes earlier than desired morning awakening (EMA) as indices of sleep duration. Additionally, sleep onset latency, sleep efficiency, number of wake after sleep onset episodes and time in bed were calculated. Self-reported sleep quality was assessed using the 5 point Likert scale incorporated into the DSD ${ }^{19}$.

\section{Statistical Analysis}

For baseline characteristics, mean (SD) for continuous variables and percentages for categorical variables were calculated. After removing the extreme outliers, we fitted linear regression models to predict MEC and HEI with sleep indices as predictors. Finally, five models were performed in multiple regression analysis. These models were adjusted for age, gender, education level, and body mass index. The results from regression analysis are presented as $\beta$-coefficients (standardized/unstandardized) with $p$ values. In Model 1 we included energy consumption as predicted by total sleep time. In Model 2 we included energy consumption as predicted by time in bed. In Model 3, we included energy consumption as predicted by early morning awakenings. In Model 4, we included energy consumption as predicted by sleep quality. In our final Model 5, we included energy consumption as predicted by all sleep indicies combined from other models. The level of statistical significance for all models was set at 0.05 . To test the robustness of the analysis, we conducted a sensitivity analysis by excluding 3 participants who did not completed the DSD at least four times. All statistical analyses were done using STATA version 11 (StataCorp, LLC, College Station, TX, USA).

\section{Results}

The demographic and anthropometric characteristics of the study population are described in Table 1. Most participants were women $(61 \%)$ and non-Hispanic white with the remainder primarily Hispanic. On average, study participants had at least 2 years of college education and had a mean body mass index of $30.22\left(\mathrm{~kg} / \mathrm{m}^{2}\right)$

As shown on Table 1, average time in bed (TIB) and total sleep time (TST) were 541.8 minutes (9.0 hrs) and 461.1 minutes (7.6 hrs) respectively. However, the range was large with minimums of [5.7 hrs for TIB/ 5.2 hrs for TST] and maximums of [ 15.2 hrs for TIB and 10.1 for TST] respectively. Mean sleep efficiency was within normal limits (91\%). There generally were few episodes of wake after sleep onset, and only a small amount of time (14 min) spent awake in the morning (EMA). Self-reported sleep quality was 2.39 using a 5-point Likert scale from 0 to 4 representing very poor, poor, good and very good sleep respectively. 
Table 2 shows the MEC as well as the intake of protein, carbohydrates, fats, fruits, and vegetables. Normative values for men and women in the United States are provided for comparison. The MEC was below averages for national data in both men and women ${ }^{20-22}$. Absolute intake of protein and carbohydrates was higher than recommended levels but was within recommended levels as a percent of energy consumption. Absolute intake of total fat was at the higher limit of recommended levels but exceeded them as a percent of energy consumption. Consumption of fruit and vegetables were below recommendations. The HEI also was markedly reduced (Men: 43.9 \pm 8.9 ; Women: $49.9 \pm 10.9$ vs. 59 for average American diet $)^{21}$.

Univariate regression models examining the impact of age, gender, education, and BMI on MEC are presented in Table 3. Male gender was associated with higher MEC but age, education and BMI were not. Univariate regression models assessing the effect of the various sleep variables demonstrated that only TST, TIB, EMA and sleep quality significantly affected MEC (data not shown). Therefore, each of these factors were included by themselves in multivariate regression models that also incorporated age, gender, education and BMI (Table 3). Sleep quality was positively associated with MEC while EMA was negatively associated. There was no significant relationship between MEC and TST. A final model integrating TST, TIB, EMA and sleep quality showed that only sleep quality was associated with higher MEC, but EMA had no significant impact. In a sensitivity analysis, we excluded the participants who had not completed the DSD at least 4 times. However, this did not materially change the results.

Regression models were calculated to examine the impact of sleep on dietary components and the HEI. None were shown to be significant (data not shown).

\section{Discussion}

In this study of persons who recently involuntarily became unemployed, we did not find any significant associations between their MEC and various parameters related to sleep duration and sleep fragmentation. However, overall positive subjective sleep quality was associated with greater MEC. Individual dietary components and the HEI also were not related to sleep duration or fragmentation but did indicate that the diet of involuntarily unemployed persons is of lower quality, based on HEI 2015 scoring, than average for US adults. ${ }^{18} 21$

In most, but not all studies, sleep duration has been shown to be inversely associated with MEC. In contradistinction, our analyses did not find any significant relationship with respect to either TST or TIB. Although minutes spent awake in the morning before getting out of bed (EMA) was inversely associated with MEC, this association was borderline and not significant in a fully adjusted model. Similarly, sleep efficiency and number of wake after sleep onset episodes were not related to MEC. Explanations for increased MEC with restricted sleep duration or fragmentation include but are not limited to changes in the relative levels of satiety and hunger 
hormones, greater available time to eat, altered timing of meals and hedonic feeding ${ }^{23}$ Our data suggest that in this population, the impact of these factors is not sufficient enough to alter MEC. Importantly, a large body of evidence suggests under-reporting of dietary intake is associated with obesity, female sex and lower education ${ }^{24}$ and may be more common among Hispanics ${ }^{25}$ who accounted for $33 \%$ of our sample. Systematic under-reporting of intake may have undermined our ability to capture significant associations between energy intake and sleep in this study.

Subjective sleep quality was positively correlated with MEC; better sleep quality was associated with higher levels of MEC. The direction of this finding is inconsistent with previous studies that have noted better sleep quality is associated with more nutritious diets and less obesity ${ }^{26}$. The lack of agreement between daily subjective overall sleep quality, and specific individual subjective sleep quality metrics as well as objective sleep quality instruments (e.g., actigraphy) has been reported previously. In a recent study of sleep quality in older adults, the specific measures assessed by the DSD used in this study was compared to the Pittsburgh Sleep Quality Index as well as subjective sleep quality recorded in the diary. Little agreement was observed among all three measures. ${ }^{28}$ Furthermore, subjective estimates of sleep or alertness have been shown to be a poor predictor of other aspects of human behavior and performance. ${ }^{29,30}$ Our findings provide a unique perspective on the use of the DSD, an instrument that is considered a gold-standard for the assessment of sleep in persons with insomnia subject to less retrospective recall bias that global estimates of sleep quality.

We observed that the diet of recently unemployed persons differed in many categories from recommendations and guidelines made by the Institute of Medicine and the US Department of Agriculture. Additionally, the HEI of the average American is already suboptimal at 59 and mean scores appear to be even lower in our sample of unemployed individuals. Food cost is inversely correlated with diet quality and is one factor that contributes to the higher prevalence of unhealthy diets in those with lower socioeconomic status. ${ }^{31}$ Our findings extend these previous observations by demonstrating the adverse economic impact of recent job loss is associated with worse diet quality.

In conclusion, in recently unemployed persons, subjective diary assessments of sleep quality were not associated with mean energy consumption. However, the diet quality of unemployed persons was found to unhealthier than the average American and consistent with the relationship between poor socioeconomic status and lower diet quality.

\section{Acknowledgements}

The authors would like to thank the staff and participants of the Assessing Daily Activity Patterns Through Occupational Transitions Study (ADAPT). The authors would like to gratefully acknowledge the assistance of the Arizona Department of Economic Security in study recruitment, and the support of the University of Arizona Collaboratory for Metabolic Disease Prevention and Treatment. 
The ADAPT study was supported by the US National Heart, Lung, and Blood Institute (HL117995). 


\section{References}

1. Ogden CL, Carroll MD, Fryar CD, Flegal KM. Prevalence of Obesity Among Adults and Youth: United States, $2011-2014$. NCHS Data Brief 2015;219:1-8.

2. Bhupathiraju SN, Hu FB. Epidemiology of Obesity and Diabetes and Their Cardiovascular Complications. Circ Res 2016;118(11):1723-735.

3. Cheon BK, Hong YY. Mere experience of low subjective socioeconomic status stimulates appetite and food intake. Proc Natl Acad Sci U S A 2017;114 (1):72-77.

4. Baik K, Hosseini M, Priesmeyer HR. Correlates of psychological distress in involuntary job loss. Psychol Rep 1989;65(3_suppl2):1227-1233.

5. Berchick ER, Gallo WT, Maralani V, Kasl SV. Inequality and the association between involuntary job loss and depressive symptoms. Soc Sci Med 2012;75(10):1891-1894.

6. Akil L, Ahmad HA. Effects of socioeconomic factors on obesity rates in four southern states and Colorado. Ethn Dis 2011;21(1):58-62.

7. Latif E. The impact of macroeconomic conditions on obesity in Canada. Health Econ 2014;23 (6):751-759.

8. Schröder M. Jobless now, sick later? Investigating the long-term consequences of involuntary job loss on health. Adv Life Course Res 2013;18(1):5-15.

9. Parvaneh K, Poh, B., Hajifaraji, M., \& Ismail, M. Less Sleep Duration and Poor Sleep Quality Lead to Obesity. Proceedings of the Nutrition Society 2016;75(OCE1).

10. Spiegel K, Leproult R, L'Hermite-Balériaux M, Copinschi G, Penev PD, Van Cauter E. Leptin levels are dependent on sleep duration: relationships with sympathovagal balance, carbohydrate regulation, cortisol, and thyrotropin. J Clin Endocrinol Metab 2004;89(11):5762-5771.

11. Van Cauter E, Leproult R, Plat L. Age-related changes in slow wave sleep and REM sleep and relationship with growth hormone and cortisol levels in healthy men. Jama 2000;284(7):861-868.

12. Haynes PL, Silva GE, Howe GW, et al. Longitudinal assessment of daily activity patterns on weight change after involuntary job loss: the ADAPT study protocol. BMC Public Health 2017;17 (1):1-11.

13. Vasquez MM, Goodwin JL, Drescher AA, Smith TW, Quan SF. Associations of dietary intake and physical activity with sleep disordered breathing in the Apnea Positive Pressure Long-Term Efficacy Study (APPLES). J Clin Sleep Med 2008;4(5):411-418.

14. Rock CL, Thornquist MD, Kristal AR, et al. Demographic, dietary and lifestyle factors differentially explain variability in serum carotenoids and fat-soluble vitamins: baseline results from the sentinel site of the Olestra Post-Marketing Surveillance Study. J Nutr 1999;129(4):855-64. 
15. Subar AF, Thompson FE, Potischman N, et al. Formative research of a quick list for an automated self-administered 24-hour dietary recall. J Am Diet Assoc 2007;107(6):1002-1007.

16. Feskanich D, Sielaff BH, Chong K, Buzzard IM. Computerized collection and analysis of dietary intake information. Comput Methods Programs Biomed 1989;30(1):47-57.

17. Martin CK, Han H, Coulon SM, Allen HR, Champagne CM, Anton SD. A novel method to remotely measure food intake of free-living individuals in real time: the remote food photography method. Br J Nutr 2009;101(3):446-456.

18. . . (Accessed at https://www.fns.usda.gov/how-hei-scored.)

19. Buysse DJ, Ancoli-Israel S, Edinger JD, Lichstein KL, Morin CM. Recommendations for a standard research assessment of insomnia. Sleep 2006;29(9):1155-1173.

20. U.S. Department of Agriculture and U.S. Department of Health and Human Services. Dietary Guidelines for Americans, 20202025. 9th Edition. December 2020. Available at DietaryGuidelines.gov.

21. 2015-2020 Dietary Guidelines for Americans. U.S. Department of Health and Human Services and U.S. Department of Agriculture. .2015.

22. Services. USDoAaUSDoHaH. Dietary Guidelines for Americans, 2020-2025. 9th Edition. December 2020. Available at DietaryGuidelines.gov. . 2020.

23. Dashti HS, Scheer FA, Jacques PF, Lamon-Fava S, Ordovás JM. Short sleep duration and dietary intake: epidemiologic evidence, mechanisms, and health implications. Adv Nutr 2015;6(6):648-659.

24. Kye S, Kwon SO, Lee SY, et al. Under-reporting of Energy Intake from 24-hour Dietary Recalls in the Korean National Health and Nutrition Examination Survey. Osong Public Health Res Perspect 2014;5(2):85-91.

25. Bothwell EK, Ayala GX, Conway TL, Rock CL, Gallo LC, Elder JP. Underreporting of food intake among Mexican/MexicanAmerican Women: rates and correlates. J Am Diet Assoc 2009;109(4):624-632.

26. St-Onge MP, Mikic A, Pietrolungo CE. Effects of Diet on Sleep Quality. Adv Nutr 2016;7(5):938-949.

27. Ding C, Lim LL, Xu L, Kong APS. Sleep and Obesity. J Obes Metab Syndr 2018;27(1):4-24.

28. Landry GJ, Best JR, Liu-Ambrose T. Measuring sleep quality in older adults: a comparison using subjective and objective methods. Front Aging Neurosci 2015;7;166

29. Van Dongen HP, Maislin G, Mullington JM, Dinges DF. The cumulative cost of additional wakefulness: dose-response effects on neurobehavioral functions and sleep physiology from chronic sleep restriction and total sleep deprivation. Sleep 2003;26 (2):117126.

30. Zavecz Z, Nagy T, Galkó A, Nemeth D, Janacsek K. The relationship between subjective sleep quality and cognitive performance in healthy young adults: Evidence from three empirical studies. Sci Rep 2020;10:020-61627.

31. Rehm CD, Monsivais P, Drewnowski A. Relation between diet cost and Healthy Eating Index 2010 scores among adults in the United States 2007-2010. Prev Med 2015;73:70-5. 

author/funder, who has granted medRxiv a license to display the preprint in perpetuity. It is made available under a CC-BY 4.0 International license. 


\section{Abbreviations \\ SES: Socioeconomic status}

ADAPT: Assessing Daily Activity Patterns through Occupational Transitions

UI: unemployment insurance

MEC: mean energy consumption

BMI: body mass index

USDA: United States Department of Agriculture

HEI: Healthy Eating Index

DSD: Daily Sleep Diary

TST: total sleep time

EMA: earlier than desired morning awakening

SD: Standard Deviation

TIB: time in bed 
Table 1: Baseline Characteristics

\begin{tabular}{lccc}
\hline VARIABLES & $\mathrm{N}$ & Mean & $\mathrm{sd}$ \\
\hline \multicolumn{1}{c}{ Demographic/Anthropometric } & & & \\
Age (years) & 183 & 40.93 & 10.24 \\
Gender (\% Men) & 183 & 38.8 & -- \\
Ethnicity (\% Hispanic) & 183 & 33.0 & -- \\
Education & 183 & 4.027 & 1.61 \\
BMI (kg/m ${ }^{2}$ ) & 183 & 30.22 & 8.08 \\
& & & \\
\multicolumn{1}{c}{ Sleep } & & & \\
Time in Bed (min) & 183 & 541.80 & 77.55 \\
Total Sleep Time (min) & 183 & 461.10 & 56.49 \\
Sleep Latency (min) & 183 & 26.56 & 22.52 \\
Sleep Efficiency (\%) & 183 & 0.91 & 0.06 \\
Wake After Sleep Onset Episodes & 183 & 1.52 & 1.09 \\
Early morning awakening (min) & 183 & 14.36 & 24.15 \\
Sleep quality & 183 & 2.40 & 0.57 \\
& & &
\end{tabular}


Table 2: Self-reported 24-hour Dietary Intake among Recently Unemployed U.S. Adults

\begin{tabular}{|c|c|c|c|c|c|}
\hline VARIABLES & $\begin{array}{c}\text { Men } \\
(\mathrm{N}=70)\end{array}$ & sd & $\begin{array}{c}\text { Women } \\
(\mathrm{N}=110)\end{array}$ & sd & Recommended or Average Values \\
\hline Mean energy consumption (kcal) & $1,898.5^{7}$ & 654.7 & $1522.2^{7}$ & 501.2 & 2000-2400 (Men); 1600-2000(Women) ${ }^{1}$ \\
\hline Carbohydrates $(g)$ & $220.7^{7}$ & 94.1 & $175.9^{7}$ & 70.2 & 130 (Men and Women) $^{1}$ \\
\hline Carbohydrates (\% of Energy) ${ }^{8}$ & 44.9 & 11.6 & 45.1 & 9.4 & 45-65 (Men and Women) ${ }^{1}$ \\
\hline Protein $(g)$ & $78.8^{7}$ & 33.5 & $60.4^{7}$ & 19.5 & 56 (Men); 46 (Women) ${ }^{1}$ \\
\hline Protein (\% of Energy) ${ }^{8}$ & 16.8 & 5.3 & 16.4 & 4.6 & 10-35 (Men and Women) ${ }^{1}$ \\
\hline Total Fat $(g)^{8}$ & 77.7 & 30.6 & 65.0 & 26.8 & $44-77^{5}$ \\
\hline Total Fat (\% of Energy) ${ }^{8}$ & 35.4 & 8.3 & 36.9 & 7.7 & 20-35 (Men and Women) ${ }^{1}$ \\
\hline Saturated Fatty Acids $(g)^{8}$ & 26.7 & 12.9 & 22.4 & 11.4 & As low as possible ${ }^{3}$ \\
\hline Trans-fatty Acids $(g)^{8}$ & 2.1 & 1.3 & 1.6 & 1.0 & As low as possible ${ }^{3}$ \\
\hline Daily servings of Fruits (cups eq/d) & $1.3^{7}$ & $1.4^{7}$ & 0.8 & 0.7 & 2.5 (Men) 1.5 (Women) $)^{2}$ \\
\hline Daily servings of Vegetables (cups eq/d) & $2.6^{7}$ & $1.4^{7}$ & 3.2 & 1.3 & 3.5 (Men); 2.5 (Women) ${ }^{2}$ \\
\hline Healthy Eating Index & $43.9^{7}$ & 8.9 & $49.9^{7}$ & 10.9 & Highest Score: 100; Average American Diet: $59^{6}$ \\
\hline
\end{tabular}

${ }^{1}$ U.S. Department of Agriculture and U.S. Department of Health and Human Services. Dietary Guidelines for Americans, 2020-2025. 9th Edition. December 2020. Available at DietaryGuidelines.gov. Based on age $\geq 19$ years

${ }^{2}$ ibid, Based on age $31-59$ years

${ }^{3}$ Institute of Medicine 2005. Dietary Reference Intakes for Energy, Carbohydrate, Fiber, Fat, Fatty Acids, Cholesterol, Protein, and Amino Acids. Washington, DC: The National Academies Press. https://doi.org/10.17226/10490.

${ }^{4}$ 2015-2020 Dietary Guidelines for Americans. U.S. Department of Health and Human Services and U.S. Department of Agriculture.

https://health.gov/dietaryguidelines/2015/guidelines/.

${ }^{5}$ Extrapolated from $2000 \mathrm{kcal}$ diet with $20-35 \%$ fat content

${ }^{6}$ U.S. Department of Agriculture and U.S. Department of Health and Human Services. Dietary Guidelines for Americans, 2020-2025. 9th Edition. December 2020. Available at DietaryGuidelines.gov. Based on Age 31-59 years

${ }^{7} \mathrm{p}<0.001$ vs. Recommended or Average Values

${ }^{8}$ Statistical comparison not performed because Recommended Values were a range 
Table 3: Regression Coefficients Predicting Energy Consumption

\begin{tabular}{|c|c|c|c|c|c|c|c|c|c|}
\hline \multicolumn{5}{|c|}{ Univariate Analysis predicting MEC } & \multicolumn{5}{|c|}{ Multivariate Analysis predicting MEC } \\
\hline VARIABLES & Age & Gender & Education & BMI & $\begin{array}{c}\text { TST } \\
\text { Model } 1 \\
\end{array}$ & $\begin{array}{c}\text { TIB } \\
\text { Model 2 } \\
\end{array}$ & $\begin{array}{c}\text { EMA } \\
\text { Model } 3 \\
\end{array}$ & $\begin{array}{c}\text { Sleep Quality } \\
\text { Model } 4\end{array}$ & $\begin{array}{l}\text { Combined } \\
\text { Model } 5 \\
\end{array}$ \\
\hline $\begin{array}{l}\text { Total Sleep time } \\
\text { (TST) }\end{array}$ & & & & & $-0.50(-.05)$ & & & & $-0.63(-0.06)$ \\
\hline & & & & & $(0.76)$ & & & & $(0.99)$ \\
\hline Time in Bed & & & & & & $-0.69(-0.09)$ & & & $0.21(-0.03)$ \\
\hline & & & & & & $(0.56)$ & & & $(0.73)$ \\
\hline $\begin{array}{l}\text { Early Morning } \\
\text { Awakening (EMA) }\end{array}$ & & & & & & & $-3.95(-0.16)^{* *}$ & & $-3.33(-0.14)$ \\
\hline & & & & & & & $(1.77)$ & & (1.8) \\
\hline Sleep Quality (SQ) & & & & & & & & $153.02(0.15)^{* *}$ & $134.8(0.13)^{* *}$ \\
\hline & & & & & & & & $(74.59)$ & $(80)$ \\
\hline Age & -6.39 & & & & $-7.1(-0.12)$ & $-6.94(-0.12)$ & $-6.54(-0.11)$ & $-6.71(-0.12)$ & $-7.24(-0.13)$ \\
\hline & $(4.29)$ & & & & $(4.2)$ & $(4.1)$ & $(4.1)$ & $(4.05)$ & $(4.11)$ \\
\hline Gender & & $-376.4 * * *$ & & & $-401.4(-0.331)^{* * *}$ & $-381.63(-0.31)^{* * *}$ & $-377.77(-0.31) * * *$ & $-390.5(0.32) * * *$ & $-353.77(-0.29) * * *$ \\
\hline & & $(86.5)$ & & & $(87.4)$ & $(89.1)$ & $(86.87)$ & $(86.45)$ & $(88.8)$ \\
\hline Education & & & 37.21 & & $55.3(0.15)^{* *}$ & $50.64(0.14)$ & $44.84(0.12)$ & $44.91(0.12)$ & $35.88(0.09)$ \\
\hline & & & $(27.5)$ & & $(26.4)$ & $(26.6)$ & $(26.5)$ & (26.6) & $(26.9))$ \\
\hline BMI & & & & 0.99 & $4.03(0.05)$ & $3.76(0.05)$ & $6.05(0.08)$ & $5.88(0.08)$ & $6.39(0.09)$ \\
\hline & & & & (5.6) & $(5.38)$ & (5.36) & (5.33) & (5.34) & (5.39) \\
\hline Observations & 180 & 180 & 180 & 180 & 180 & 180 & 180 & 180 & 180 \\
\hline R-squared & 0.01 & 0.09 & 0.01 & 0.0002 & 0.13 & 0.14 & 0.16 & 0.15 & 0.18 \\
\hline
\end{tabular}

Standard errors in parentheses

$* * * \mathrm{p}<0.01, * * \mathrm{p}<0.05, * \mathrm{p}<0.1$ 
Model 1: Mean energy consumption as predicted by total sleep time (TST)

Model II: Mean energy consumption as predicted by time in bed (TIB)

Model III: Mean energy consumption as predicted by early morning awakenings (EMA)

Model IV: Mean energy consumption as predicted by sleep quality (SQ)

Model V: Mean energy consumption as predicted by TST, TIB, EMA, and SQ. 